Revue de droit comparé du travail et de la sécurité sociale

3 | 2017

Le travail dans l'économie informelle, un défi pour le droit social

\title{
La compensation équitable pour licenciement individuel
}

Nicola Gundt

\section{OpenEdition}

\section{Journals}

Édition électronique

URL : https://journals.openedition.org/rdctss/456

DOI : $10.4000 /$ rdctss.456

ISSN : 2262-9815

Éditeur

Centre de droit comparé du travail et de la sécurité sociale

Édition imprimée

Date de publication : 1 septembre 2017

Pagination : 220-221

ISSN : $2117-4350$

Référence électronique

Nicola Gundt, «La compensation équitable pour licenciement individuel », Revue de droit comparé du travail et de la sécurité sociale [En ligne], 3 | 2017, mis en ligne le 01 novembre 2017, consulté le 04 décembre 2021. URL : http://journals.openedition.org/rdctss/456 ; DOI : https://doi.org/10.4000/ rdctss. 456

\section{(c) $\Theta \Theta \Theta$}

Revue de droit comparé du travail et de la sécurité sociale est mise à disposition selon les termes de la Licence Creative Commons Attribution - Pas d'Utilisation Commerciale - Pas de Modification 4.0 International. 


\section{NICOLA GUNDT Maastricht University}

\section{LA COMPENSATION ÉQUITABLE POUR LICENCIEMENT INDIVIDUEL}

1. La Cour de cassation des Pays Bas s'est pour la première fois prononcée sur la nouvelle « compensation » équitable en cas de licenciement étant entendu que la loi ne définit pas s'il s'agit d'une indemnisation ou d'un dédommagement.

Depuis juillet 2015, le droit du licenciement néerlandais connaît deux types de compensation (vergoeding). Le premier est une indemnité fixée par la loi, payable en cas de cessation d'un contrat de travail d'une durée d'au moins 24 mois alors qu'aucun reproche sérieux ne peut être imputé au salarié. Le but de cette indemnité fixe est double : elle compense la perte de travail et en même temps elle facilite la recherche d'un nouvel emploi, d'où le nom d'indemnité transitoire (transitievergoeding). Selon le législateur, avec cette indemnité calculée selon le mode défini par l'article $7: 673$ du Code civil, toutes les conséquences d'un licenciement «normal » sont compensées.

Le deuxième type de compensation est dite «équitable ». Elle doit être versée dans des circonstances spécifiques, par exemple si l'employeur a agi contrairement à la loi, si son attitude est sérieusement répréhensible et en cas d'appel ou de cassation. Ce sont les deux premières hypothèses que nous retiendrons ici. Le concept d'une compensation pour comportement fautif est quelque chose de nouveau. Malheureusement, le concept n'a pas été clarifié lors des travaux préparatoires de la loi ou lors des débats parlementaires. On sait seulement qu'il ne s'applique que dans des cas très graves et exceptionnels. La compensation est due en raison du comportement extrêmement blâmable de l'employeur. Elle a, en plus, pour fonction de prévenir de tels comportements.

2. En raison du manque de clarté du législateur concernant cette compensation équitable, doctrine et jurisprudence essaient de produire, sinon clarté, du moins un débat. Ce débat comprend deux principaux volets. Le premier porte sur la frontière assez vague entre le comportement simplement blâmable et le comportement sérieusement répréhensible. C'est pourquoi, on dispose de jugements relativement peu convaincants, comme celui d'un enseignant qui donnait les réponses aux questions d'un examen aux élèves. Ce comportement, selon le juge, n'est pas sérieusement répréhensible, car l'enseignant n'a rien gagné avec cette action.

Le deuxième volet du débat concerne le caractère et le mode de calcul de cette compensation. Ici encore, il faut souligner le manque de clarté du législateur pour savoir si la nouvelle compensation équitable devait être considérée comme l'équivalent d'un dédommagement pour licenciement manifestement injustifié. Dans ce cas, les conséquences du licenciement pour le salarié sont décisives. Après juillet 2015, par contre, les conséquences du licenciement sont compensées par une indemnité fixe. Mais alors quelles sont les conséquences qui doivent être indemnisées par la compensation équitable?

3. L'incertitude et le manque de précision sont à l'origine de vives discussions doctrinales mais aussi entre juges. La doctrine paraît désormais unanime pour ce qui concerne cette compensation. On pense que, comme avant 2015, il faut un double test. En premier lieu, il faut établir si le comportement de l'employeur était vraiment sérieusement blâmable. Ce n'est que dans l'affirmative que la deuxième question sur la compensation se pose. Concernant cette deuxième question, la doctrine plaide pour un calcul selon deux principes. Premièrement, la compensation devrait être rapprochée du comportement sérieusement répréhensible de l'employeur ; plus précisément, il s'agit de mesurer le degré de gravité du comportement. Deuxièmement, la doctrine soutient que le montant de la compensation soit au moins égal 
au montant du salaire que l'employeur aurait dû payer si le salarié avait demandé l'annulation de la décision du licenciement au lieu d'exiger des compensations.

Toutefois, des auteurs interprètent les travaux préparatoires et les débats parlementaires en vertu desquels la compensation équitable doit avoir un caractère punitif. En effet, le législateur défendait que la compensation devait aussi permettre de prévenir les comportements fautifs de l'employeur. Mais est-ce la même chose que les dommages punitifs?

4. Les jugements en première instance et en appel donnent aussi une impression d'incertitude, voire d'une certaine désorientation. Il existe trois types de jugement. Le premier comprend des jugements qui accordent une compensation équitable sans aucune motivation ou élucidation du mode de calcul ou des aspects pris en considération. Le problème posé par ces jugements est que le salarié n'a aucune idée des motivations et des arguments du juge. Avant 2015, cela ne faisait pas problème parce qu'il n'était pas possible de se pourvoir en appel. Depuis 2015, le droit à l'appel est fixé à l'article $7: 683$ BW. C'est pourquoi les motivations des décisions sont désormais plus importantes.

Le deuxième groupe de jugements concerne les juges qui accordent une compensation plus ou moins égale au salaire dû au salarié s'il avait opté pour l'annulation de la décision de licenciement et donc pour la continuité du contrat de travail avec droit au salaire. Pour ces juges, l'argument principal est que, l'action en annulation et l'action pour compensation équitable étant équivalentes, la compensation devrait être plus ou moins égale.

Le troisième groupe de décisions fixe le montant de la compensation équitable à la faute commise par l'employeur pour le punir et prévenir un tel comportement.

C'est dans de telles circonstances que la Cour de cassation devait juger un cas de compensation équitable. Le litige concernait une coiffeuse licenciée à cause d'un différend sur les dates de congés. Elle demandait une compensation équitable en plus de la compensation fixe. Les juges en première instance et en appel ont alloué cette compensation. En cassation, la coiffeuse estime que le mode de calcul de cette compensation n'était pas correct dans le sens où la compensation étant de nature punitive elle devait au moins être égale au montant du salaire dû en cas d'annulation de la décision de licenciement.

La Cour de cassation commence par avancer une explication brève du système des compensations rappelant que l'indemnité fixe vise à compenser les conséquences d'un licenciement tandis que la compensation équitable, au contraire, n'est due que dans des cas extrêmes. Regardant le mode de calcul et les aspects devant être pris en considération, la Cour de Cassation offre une ébauche de raisonnement. Comme la compensation équitable est liée à la gravité du comportement fautif de l'employeur, seules les conséquences directement liées au dit comportement sont prises en considération. En plus, la Cour partage l'idée selon laquelle l'action en annulation de la décision de licenciement et la demande de compensation sont équivalentes. Ainsi, en calculant le montant des compensations, le juge doit faire prendre en compte les salaires que l'employeur aurait dû verser si le salarié avait demandé l'annulation du licenciement. Concernant la possibilité de verser des salaires futurs, le juge doit décider si la volonté du salarié de ne pas poursuivre la relation de travail mais de demander une compensation a pour motif le comportement de l'employeur. D'un autre côté, si le salarié a déjà trouvé un autre emploi, cela peut aussi avoir une influence sur le montant de la compensation. Pour conclure, la Cour de Cassation a décidé que le salarié doit être dédommagé pour les fautes commises par l'employeur et qu'un licenciement contraire à la loi ne doit pas être plus facile ou moins onéreux qu'un licenciement réalisé en toute légalité. Cependant, rien ne dit ce que signifie la notion de dommages punitifs. Certes, l'employeur doit ainsi être dissuadé de se comporter de la même façon; mais cela ne doit pas être compris comme étant un droit à un dédommagement à caractère punitif. 